\title{
Integrating Semantic Web Services into Financial Decision Support Process
}

\author{
Ilona Pawełoszek \\ Częstochowa University \\ of Technology, \\ Faculty of Management \\ Poland \\ Email: ipaweloszek@zim.pcz.pl
}

\begin{abstract}
The operating environment of Small and Mediumsized Enterprises (SMEs) is more uncertain and risky comparing to big enterprises. The SMEs managers often face insufficient funds and lack of domain expertise to undertake important financial decisions. Moreover they are often unaware of opportunities offered by financial institutions. Therefore there is a growing need for intelligent and interactive webbased tools integrating data and providing information from heterogeneous sources, oriented on risk-detection and decision support for small and medium enterprises. In this paper the semantic Web services platform has been proposed, which integrates the internal data from the company's databases and external Web resources to perform dynamic evaluation of the financial position of enterprise. The designed platform detects signals indicating the need for action, and it composes a process choosing available Web services aiming at improving the company's financial situation.
\end{abstract}

$\mathrm{C}$ OMPETITIVE challenges on a global market require decision makers of small and medium enterprises (SMEs) to have up-to-date and relevant knowledge of the economic situation of the company and its environment. Managers must have the possibility to identify and analyze all indicators that may have impact on the operations of the enterprise and moreover they have to react to market changes and take the appropriate actions. In a world teeming with overwhelming amount of data accessible by the World Wide Web analyzing information becomes very difficult. Discovering and understanding all dependences between various financial ratios while taking into account economic trends is crucial because they alert one to anomalies and threats [1].

Unfortunately, many of the managers of the SMEs lack expertise or meaningful experience in financial domain as well as the potentials the Web services can bring to the business. Furthermore, SMEs operate in a definitely more uncertain and risky environment than big enterprises. A complex and dynamic market changes have much more impact on SMEs' financial situation than on big companies. Tolerance of mistakes is narrower [2], [3]. Usually financial expertise is either not available or too expensive for the managers of SMEs. Big companies, in contrast, have at their disposal strategic consultation and elaborated procedures to solve problems. The SMEs cannot achieve this level of expertise due to the lack of skilled personnel and financial resources.
The decision makers often act intuitively so the rationality of their decisions may be questionable. Especially in exceptional and unusual cases intuition alone can be unreliable [4]. According to Dane and Pratt [5], the effectiveness of intuitive decision-making depends on the decision-maker's level of expertise on the subject at hand.

Therefore there is a growing need for intelligent and interactive Web-based tools integrating data and providing information from heterogeneous sources, oriented on risk-detection and decision support.

The diversity of solutions and opportunities in the field of integrating data from many sources have grown with the emergence of the semantic Web services which on the one hand provide intelligent search capabilities and on the other, they offer business process automation. The semantic Web shares many goals and issues with Decision Support Systems (DSS), e.g., being able to precisely interpret data, in order to deliver relevant, reliable and accurate information to a manager. Semantic Web technologies have been used in DSS during the past decade to solve a number of different tasks, such as information integration, sharing, Web service annotation, discovery, knowledge representation and reasoning [6].

There are a lot of publications discussing technical issues and challenges in this field. However a relatively small number of literature describes the subject from the managerial point of view, particularly: how to use Web services in an innovative way to improve the agility and efficiency of business processes and how this technology can be used to bring competitive advantage for the SMEs..

In this paper the semantic Web services platform has been proposed, which integrates the internal data from the company's databases and external Web resources to detect signals (factor changes) indicating the need for decision action, then it composes a process of available Web services aiming at improving the company's financial situation.

The work contributes to the domain of early warning systems and decision support systems.

The structure of the paper is as follows. Section 2 presents a brief state of the art of the domain of Web services and financial early warning systems. Section 3 explains the advantages of semantic Web services comparing to traditional approach. In the section 4 the components of the Web servicesbased platform for decision support are presented. In order 
to illustrate the idea of using Web services in a business process an example of financial decision-support system is presented in the section 5. In the example, a part of early warning system and financial ontology will be integrated into semantically rich business processes.

\section{OVERVIEW OF THE LITERATURE}

Semantic Web services (SWS) have been extensively discussed in the literature in many contexts [7], [8]. The theoretical benefits of semantics as well as their potential impact on operational management are well known concepts. Semantic Web solutions integrated with the company's IT systems may support managers in composing the instances of business processes. However the automation is usually possible in streamlined processes with limited number of alternative flows.

In the research related to Web services, several platforms and languages have been presented that allow easy integration of heterogeneous systems. In particular Universal Description, Discovery, and Integration (UDDI) [9], Web Services Description Language (WSDL) [10], Simple Object Access Protocol (SOAP) [11] and REST [12], which define standard ways for service discovery, description and invocation. There are other initiatives such as Business Process Execution Language for Web Service (BPEL4WS) [13] and DAML-S Service Model [14] that are focused on service compositions where a flow of a process is known a priori.

There is a growing consensus that simple functional descriptions of Web services are not sufficient to develop intelligent and dynamic processes, characterized by the high degree of heterogeneity, autonomy, and distribution of service providers on the Web [15]. Due to the increasing availability of Web services that offer similar functionalities with different characteristics there is a need for more sophisticated discovery processes to match user requests [16].

The key issue to address is modeling of composition of Web Services, which should be integrated into real business processes. The focus is on the activities of discovery and selection that are required to identify the relevant Web services and to include them in a business process [17]. The idea is that a number of SWSs can meet some basic requirements specified by a manager. Then, the manager needs to be supported in choosing which one of the above SWSs better fulfills his needs. The selection and invocation of the best (according to a set of criteria) service can be automatic, or left to the discretion of a human requester [18].

Ontologies are increasingly used in describing complex data, processes and services on the Web. Here the concept of using semantics to support decision making has been proposed with particular emphasis on early warning models. There are a lot of methods and techniques of early warning and warning forecasting models that can be used by the managers of SMEs. The choice of the method is an open question because there is no synthetic indicator that would aptly describe the financial condition of the company. The prospecting works illustrate models of [19]: E.I. Altman, M. Tamari, R.J. Taffler, M. Blum, S. Appetiti, R. Edminster, E.B. Deakin, M. Zmijewski, W. H. Beaver.
Most of the aforementioned methods generate a warning signal informing mainly about threats inside the company, but also about unnoticed opportunities for further development of the company. Therefore it can be a single information or a set of information thanks to which one can predict future threats in the firm's development.

Currently organizations have a growing need for intelligent systems that can assist managers by gathering and analyzing information, making recommendations, supporting business decisions, and implementing business workflows [20]. Therefore interoperability and transparency of the technology are very important, so the managers could concentrate on the substantial aspects of the decision making process.

As the surveys conducted in different countries show [21], [22], [23], many managers of small businesses do not have grounded financial knowledge, many of them are selftaught, learn from an accountant, a consultant, or a bookkeeper. Today many important financial services companies use Web APIs to offer their customers, their staff, and their business partners new tools that streamline operations. Therefore it is possible to harness the exposed Web services to compose a process or a few variants of processes that fulfill the company's current needs. The proposition presented hereby is based on ontologies as the means of knowledge representation and interpretation of current company's financial situation. The possible actions that can be taken are suggested on the base of accessible financial Web Services. The services can be dynamically composed into the company's processes.

The next section presents the advantages of using semantic Web services in contrast to the traditional Web services approach.

\section{II.FROM TRADITIONAL TO SEMANTIC WEB SERVICES}

In traditional scenario Web services initially composed by the developer using Business Process Execution Language for Web Service (BPEL). Currently, the services are discovered by matching the service request parameters to the predefined keywords in service descriptions. This means that the process is known a-priori without any possibility to choose the best from some alternative process flows.

Semantic Web services are discovered by high level match-making techniques [24], whereas non-semantic Web services discovery methods use information retrieval techniques [25] based on keyword matching.

The traditional solution has serious limitations due to natural language descriptions, which may or may not be available and their analysis requires human intervention. The service publishers sometimes leave the service descriptions blank in the UDDI registries. In such a case even keyword matching based approach does not work.

To address the syntactical limitations of business processes and services the semantic enhancement of Web services descriptions with ontologies can be a solution. Semantic Web services enable a dynamic composition of Web services. In this case, Web services are composed at run-time: the participants of a composite Web service are discovered 
dynamically based on a variety of concerns: availability, load-balancing, cost, quality of service (QoS), etc. [26 p.251].

According to a definition by Moreau et al. [27] Semantic Discovery is the process of discovering services capable of meaningful interactions, even though the languages or structures, of their descriptions may be different. Moreover, since ontologies and Web services are developed independently the service request and advertisement can be annotated with multiple ontologies, thus facilitating better efficiency [28].

In the Web services based DSS complex scenario, it is difficult to find specific Web services to meet the requirements of the decision makers. The situation becomes even more complicated when there is no single Web service which could satisfy all of the requirements but it can only be achieved with a combination of several Web services [29].

Many business applications are built on the base of different Web services available on the Internet. These applications are highly dependent on discovering relevant and efficient web service. The discovered Web service must match with the input, output, preconditions, effects and service quality parameters specified by the user. Modeling of composition of Web services is based on algorithms for comparing available services with identified business process requirements.

Web services developed by different vendors are usually published on the Internet using Universal Description, Discovery and Integration (UDDI) [30] which are XML-based registers of services. Search in UDDI is based on keyword matching which is not efficient as huge number of Web services may match a keyword and it is difficult to find the right one. Modern approaches take advantage of semantic Web concept where Web service matching is done using ontologies. Discovering Web services automatically without manual work is an important concern [31].

Web services can be developed using different protocols, and tools. The chosen standard implies the way the service can be discovered and invoked. There are several application programming interface (API) standards in use, of which the most current is Representational State Transfer (REST) [12]. REST is a network architecture paradigm relying on standard transport protocols like HTTP, without the use of an additional messaging layer. A service call is handled via its URI. "REST provides a set of architectural constraints that, when applied as a whole, emphasizes scalability of component interactions, generality of interfaces, independent deployment of components, and intermediary components to reduce interaction latency, enforce security, and encapsulate legacy systems" [32]. Services developed in accordance with the REST paradigm are often called RESTful services. The World Wide Web is the key example of the REST design.

REST has a lower barrier to entry versus other approaches such as SOAP, RPC or CORBA. As a programming approach, REST is a lightweight alternative to the aforementioned solutions in terms of complexity of coding. Another advantage is good performance and simplicity of HTTP used to make calls between machines. A single URL is enough to call a service and the HTTP reply is the raw result data not embedded inside any code so it can be directly used without the need of parsing. Moreover the RESTful services can be invoked using a Web browser alone so it is easy to write and test applications.

However there is no standard way to publish and discover REST services (such as Web Services Description Language (WSDL) in SOAP approach where all the service descriptions are stored in a centralized UDDI registry). Many attempts were made in order to resolve this issue (the popular ones are WADL and RAML projects), but none of them has been commonly accepted by the community of REST developers. Therefore there is a challenge of adding semantics to the RESTful services [33] and it is still an active field of research and development.

Companies can benefit from exploiting semantic Web services in many aspects of their business. On the strategic level, the SWS give the possibility to build highly maintainable applications and profit from a loosely coupled architecture which facilitates the cooperation between the company and its business partners. On a tactical level, a company may reuse the ontologies that have been created for SWS in the other areas such as knowledge management and Business Intelligence. On the operational level the company can benefit from streamlined operations and data integration.

\section{COMPONENTS OF THE PLATFORM FOR SUPPORTING FINANCIAL DECISION-MAKING}

The World Wide Web allows managers to access the information from the large database repositories globally. In general the information can be used to reduce the risk of financial decisions. The large amount of information (some examples are provided later in Table II) and diversity of structures of data sources make it difficult to find the right information, therefore semantic Web technologies are crucial to improve the efficiency of discovery, automation, integration and reuse of data. Semantic Web technologies also provide support for interoperability problem which cannot be resolved with Web technologies of the previous generation.

The overall framework of the semantic Web servicesbased decision support system can be schematically illustrated on Figure 1. The source of information for business processes are transactional systems such as ERP, data warehouses, BI and the environment of the company. The external information is imported into the system by the means of Web services, which are dynamically discovered on the Internet according to the criteria defined in the business process models. The information from the internal and external sources is combined and presented to the manager to support the decision making process and to suggest alternatives for the decision maker to consider.

However the broad scope of Web services and opportunities offered by Web financial platforms creates a possibility to automate the process of consulting and decision making. The schema of the process of decision making is presented on figure 2 . 


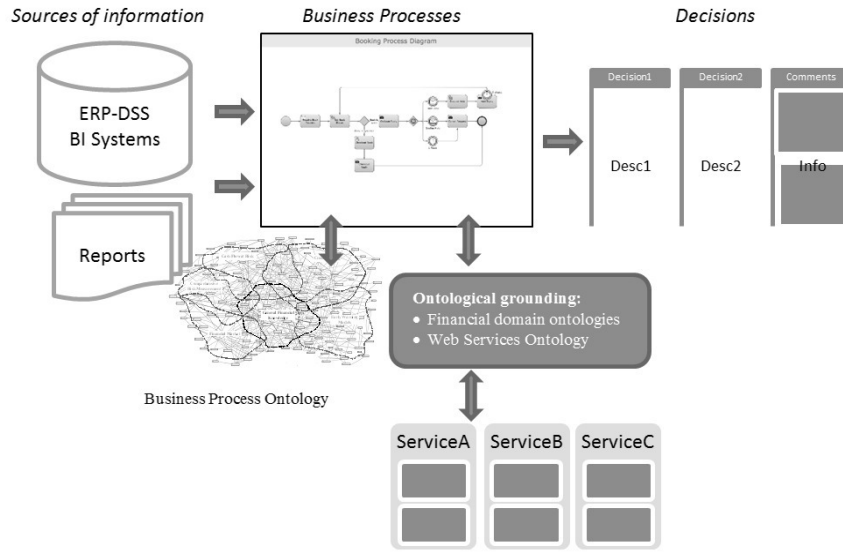

Fig. 1 Functional components of the platform

The process depicted above is written in human-readable BPMN syntax, but it can be translated into an executable BPEL process. That is not simple because of conceptual mismatch between the two process modeling languages which differ in their expressive power [34].

Some of the activities in the process can be executed automatically by discovering, selecting and invoking a proper Web service. This steps in the process diagram are marked with the sprocket icon (in BPMN) which means automation by calling the Web service (or set of Web services) that are able to perform an action predefined in the diagram.

The request for the financial data for the analysis can call the services which pull out the data from the enterprise systems and external Web services that provide data about market (for example stock prices of competitors, annual earnings, prices of products or other financial ratios). The data provided that way can be then used to further analyses and interpretations of KPIs (Key Performance Indicators), which is the next step in the process. Extracting the data from external resources offering RESTful services can be done in multiple ways. There are many existing conceptual models for service descriptions such as: SAWSDL, WSMO-Lite, hRESTS or Swagger.

In order to reduce the amount of manual effort required for finding, combining and using services, it is necessary to provide a common vocabulary. An interesting and well documented approach to provide common semantic model of Web services is the Minimal Service Model (MSM). Driven by the idea of Semantic Web and Linked Data MSM is written in RDFS. The Minimal Service Model defines services as having a number of operations, each of which have input and output messages and faults. Web APIs are also supported through the definitions of URI template, and the HTTP method [35 p.246].

Having the framework for semantic description of Web services the next thing is to build the database of instances referencing to the Web services that can be potentially useful in the financial Decision Support System.

In the case of traditional SOAP-based architectures it is quite easy to find the services because they are described and published in WSDL files which are indexed by popular search engines. The service discovery can be done for example by using Google with the search parameter ".... filetype:wsdl". Also the registers (UDDI) can be used (e.g. SeekDa.com or ServiceFinder) that crawl the Web for WSDL files. However as far there is no straightforward unified way to find RESTful Web services because it is hard to distinguish between URI of RESTful Web service and other Web resources (e.g. Web sites URLs).

The most accurate way to find RESTful services is to browse the web pages of their providers. The well-known global data providers that may offer financial services or economic data that can be used to support managerial decisions are for example: Quandl, Xignite, Bloomberg, online banks and other financial institutions .

The mentioned Minimal Service Model ontology can constitute a data structure for a triple store (RDF database such as Virtuoso) of instances. The advantage of this RDF-based solution is its flexibility and openness for adding new data structures describing new frameworks of services description. Moreover the triple stores offer exhaustive searching capabilities by the possibility to use semantic query languages (e.g. SPARQL).

The next issue is to dynamically embed the selected services into the business process definition.

As it was mentioned before there are a few points in the process flow where the services can be used: to gather data needed for the assessment of the company's financial situation and for suggesting possible alternatives (selected Web services that are relevant) for decision makers.

Because business process descriptions are constructed in their specific modelling languages such as BPMN or BPEL there is a need to do semantic mapping between the process descriptions and the services from the RDF database. For example the REST URL to get StockQuotes of 5 companies

(Microsoft, Inter Corporation, SODR Gold Trust, Silver Wheaton Corp Common Shares and MarkWest Energy Partners) may be the following:

https: / /www. westwind.com/WestWindWebToolkit/Samples/Rest/StockService.ashx?Method=GetStockQuotes\&symbolList=msft, intc, gld, slw, mwe

As it can be seen there are some terms in the URL that can be ambiguous for automatic recognition and mapping to the terms used in the business process model. For example in business process model an activity could be named: "Compare the stock prices of the competitors". This is compound activity that should invoke a few services to provide required data. The above REST request on stock quotes of 5 companies could be used. So there is a need to "know" that GetStockQuotes is one of the tasks in the considered activity.

Also the abbreviations of the companies' names can be ambiguous (there can be full names used in the process model) so there is a need to provide a dictionary / thesauri of the names and abbreviations. The components of the proposed solution are illustrated on Fig. 3. 


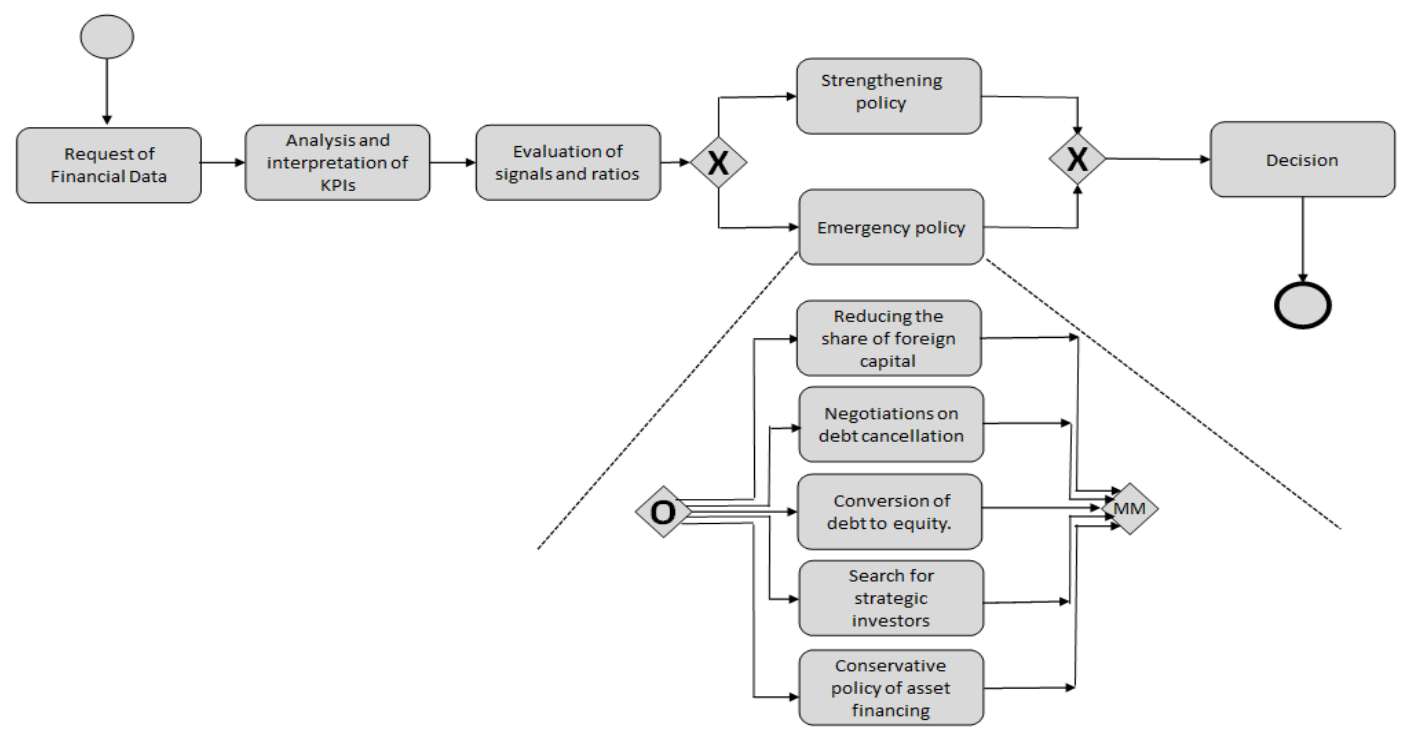

Fig. 2 The BPMN diagram of the process of financial decision-making

Irrespectively of the approach chosen (RESTful or SOAP-based Web services) the functioning of the platform can be described in the following steps:

1. The manager defines goal and requested QoS parameters

2. The system checks for available services (exposed by B2B partners, administration institutions, banks etc.). The ontology is used to identify the services that are relevant to the manager's request.

3. The SWS system extracts data about the user from IT Systems.

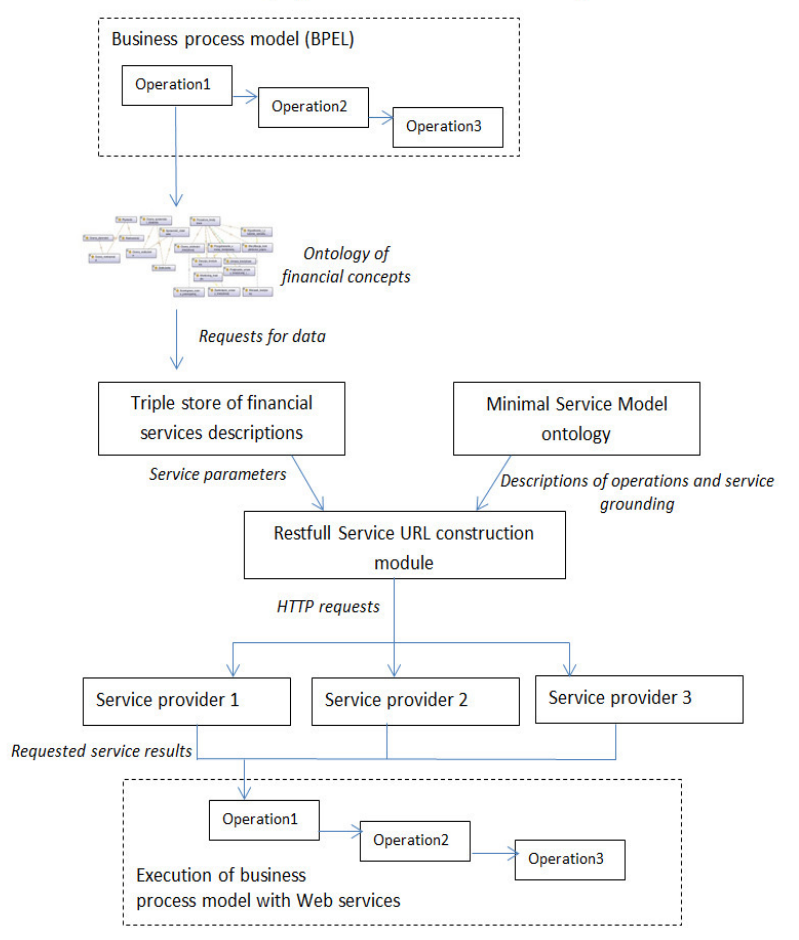

Fig. 3 The platform components with data flow
4. Reasoning capabilities are used to rate the services. In this step the user's profile and requirements are considered.

5 . Highest rated services are chosen and are recommended for the manager.

6 . The user receives offers ordered by their ratings.

7. The user can decide on the best way to perform a process. The manager can refer to the financial ontology at any time there is a need for additional explanations.

8. The corrections to the ontology can be made on the base of the manager's feedback.

The set of ontologies describing concepts, relations and functions related to the assessment of economic situation of the company was used. In the example described hereby, particularly the ontology of early warning signals pointing to financial difficulties were taken into account. The ontology facilitates the interpretation of warning signals by the manager. A part of the ontology has been presented on Fig. 2.

While conducting the analysis the manager has access to a domain ontology that includes concepts associated with models and methods of early warning and notions related with data from financial reports. These concepts can be linked with their instances - values in data bases, reports and documents to be used to determine the warning signals . The manager is supported by financial ontology while evaluating economic situation of the enterprise. The ontology helps to interpret the data coming from the ERP system, for example to determine and interpret ROA, ROE and many other ratios. The fragment of this ontology is illustrated on Fig.4.

The business processes depicted as a diagram in the center of Fig. 1 in the real scenario are defined in Business Process Execution Language - BPEL (also WS-BPEL, BPEL4WS) . BPEL provides the possibility to invoke Web services in a predefined sequence. Each business process defined in BPEL can be exposed as a Web service and included into other processes. 


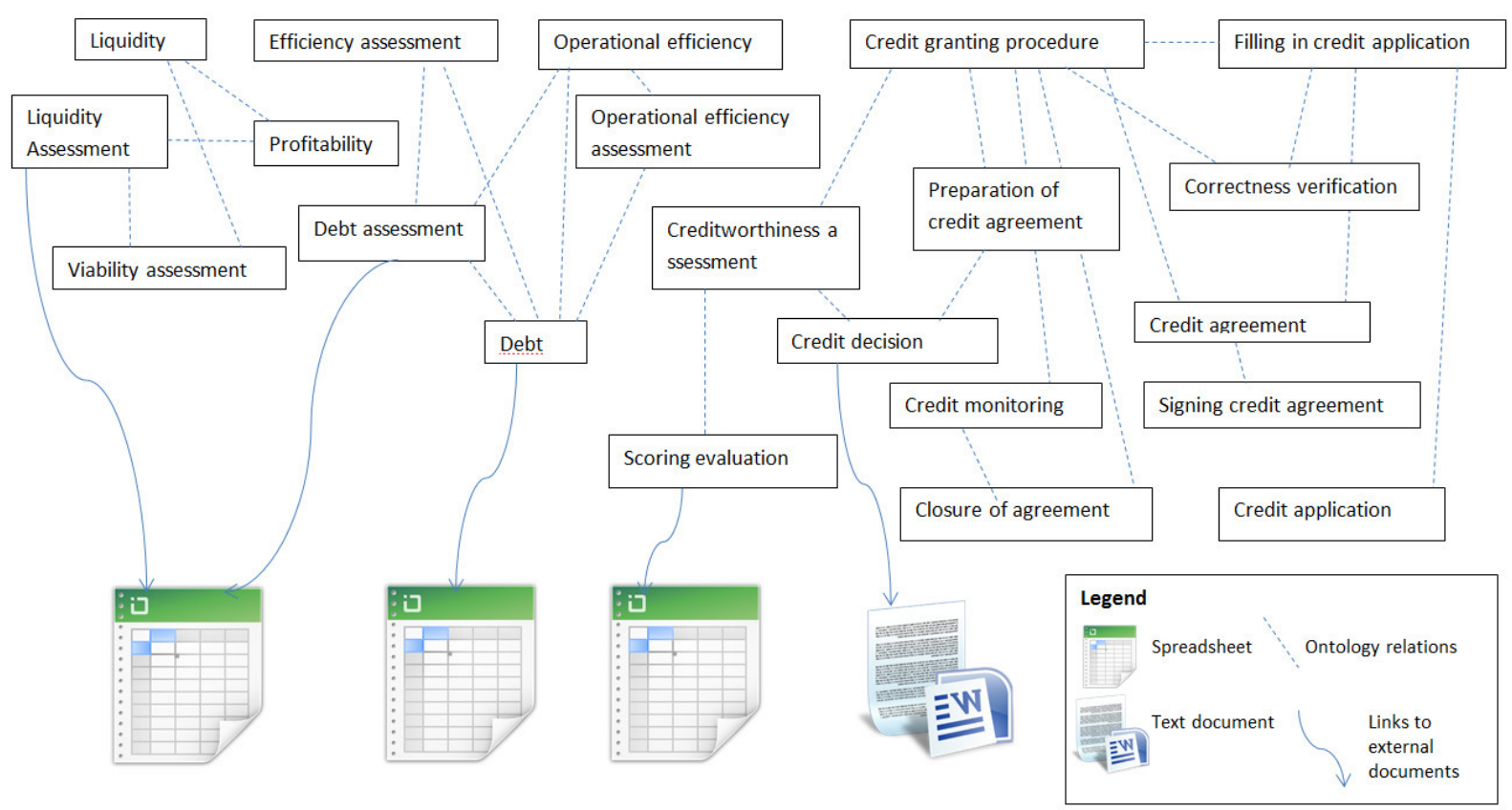

Fig. 4 Fragment of financial ontology with links to external documents

BPEL is using WSDL [36] descriptions to identify partner services, i.e. services are identified by port types and operations in the process models. As a result only services that implement a concrete interface can be used which is a major deficiency of BPEL [37].

Semantic Web Services (SWS) aim at eliminating this deficiency by automation of the development of Web service based applications through the use of ontologies. By providing formal descriptions with well-defined semantics, SWS facilitate the machine interpretation of Web Service - functional and not functional - properties. Significant results in the field of SWS are already available, in terms of reference ontologies, e.g. OWL-S [38] and WSMO [7]. The two ontologies represent different approaches to specify semantic information for Web services in order to enable automatic service discovery, composition and execution.

The OWL Profile ontology contains semantically enriched information about Web service and it is used as both an advertisement for the service and as a request to find a service on the basis of matching semantics. A first significant difference between the two approaches is that OWL-S does not separate what the user wants from what the service provides. In WSMO there is a property referred to as a goal, which specifies what the user wants and the Web service description defines what the service provides through its capability [39].

Common underlying idea behind all the semantic approaches is the dynamic selection, composition and mediation - on the basis of available semantic descriptions - of the most adequate Web resources (services and data) to accomplish the specified processes [40]. Current research efforts are investigating how SWS can be effectively applied in real world business scenarios and integrated with enterprise information systems.
It is easy to see the benefits of semantics in many application areas. For example in financial domain, a major area of financial business is the provision, selling, and management of mortgages. This is a compelling business: the provider (i.e. the bank) earns money by selling a mortgage contract, and the client is naturally interested in the cheapest offer that suites his needs. Usually, clients consider several offers and compare these in detail with respect to the monetary amount in question. For example, if several banks provide their mortgage offers via semantically described Web services, we can easily imagine an application that compares the offers and automatically selects the one which is most suitable for the customer's needs [7 p.168].

\section{EXAMPLE SCENARIO OF FINANCIAL DECISION-MAKING}

Semantic Web services can be used in many ways in an enterprise architecture, the most typical examples include supply chain management and business integration among multitude applications. One of the main advantages of SWSs is their reusability. The composition of new services by dynamically invoking the existing SWSs creates the possibility to perform very complex tasks in business processes. However, in this section the simple example of early warning system was chosen to illustrate the concept of semantic Web services integration with business processes.

The financial signals are the most popular and the broader group of quantifiable signals. They are generated on the basis of deviations from desired single values (or groups of values) of financial parameters or ratios. Selected warning signals designated on the basis of specific financial parameters are presented in Table I. It should be noted that these are example signals and their interpretation related to different areas of finances. 
TABLE I.

EXAMPLE CRITERIA OF ASSESSMENT OF FINANCIAL CONDITION OF THE ENTERPRISE

\begin{tabular}{|l|l|l|l|}
\hline \multirow{2}{*}{ Ratio } & \multicolumn{3}{|c|}{ Evaluation criteria } \\
\cline { 2 - 4 } & \multicolumn{1}{|c|}{ Good } & Average & \multicolumn{1}{c|}{ Bad } \\
\hline Asset Turnover & $>.40$ & $.25-.40$ & $<.25$ \\
\hline Current Ratio & $>1.5$ & $1.0-1.5$ & $<1.0$ \\
\hline Debt to Asset & $<.30$ & $.30-.50$ & $>.60$ \\
\hline Operating Expense & $<.65$ & $.65-.80$ & $>.80$ \\
\hline Operating Profit Margin & $>.15$ & $.05-.15$ & $<.05$ \\
\hline Return on Assets & $>.10$ & $.50-.40$ & $<.05$ \\
\hline
\end{tabular}

To illustrate, the case study concentrates on the values of ratios which indicate bad situation of the company. Moreover, it has to be stressed that during the analysis of the financial situation of the company many other ratios can be used. It is easier to determine potential problem areas when industry benchmarks are available, they can be provided by many Web portals (such as: finance.google.com, finance.yahoo.com, bizminer.com, bizstats.com).

The rank of generated warning signals can differ. Let us consider the case of using semantic services related to so called strong warning signal in the area of funding structure. This signal means high probability of financial difficulties resulting i.e. in bankruptcy.

To solve the problem the manager should take appropriate decisions associated with:

- reducing third party equity,

- negotiations on the cancellation of debt,

- the debt-to-equity swap,

- searching for strategic investors.

- conservative policy of financing current assets.

Currently financial advisory consultants offer their services to address the aforementioned problems. Their work is to find and suggest solutions and the best alternatives.

As it was mentioned before, Web services can be implemented also for taking actions as the response to the early warning signals and to put into practice the emergency policy. Ontology of financial knowledge is the foundation of the described platform. The comprehensive financial ontology takes on the role of the consultant - on the one hand it helps the manager to take decisions by improving the efficiency of analysis and increasing the capacity of understanding of financial data, and on the other hand it is needed for automatic discovery and selection of proper Web services which provide the managers with current data and financial services.

The activities included in the emergency policy are defined in the financial ontology. For example the warning signals may suggest negotiations on debt cancellation or reduction. The activities aiming at this goal may include looking for debt consolidation loans, so the system may find Web services of banks that offer debt consolidation loans and send requests to the banks' Web APIs. The requests would include parameters such as the amount of the company's debts and the deadlines of payment. Then the system can choose the best offers of banks, present them to the manager. After the manager's decision the system sends needed information to the selected bank to make finance agreement.

It is worth noticing that the aforementioned suggested actions would have strategic implications if implemented, so the decision to a high degree depends on how the given company views and solves important issues. There are many behavioral issues (such as risk aversion of the managers) that influence the choice of strategic actions. These issues cannot be resolved automatically, but the role of the proposed system is to present alternative ways of action.

The operations management problems are more relevant for automatic (or semi-automatic) handling, so the usability of the proposed system can be illustrated. The example of such problem can be liquidity analysis. The term refers to an enterprise's state of financial health. Liquidity is the ability of an organization to meet its short-term financial obligations. This issue is a major source of concern for small and medium business managers as bank loans are becoming too expensive to maintain.

Liquidity management encompasses the management of cash balances, including short-term funding and investments for excess cash. The alternatives for raising cash during temporary shortages and the opportunities to invest excess cash on a short-term basis are important to the functioning of an organization [41].

In the early-warning system the data for calculating liquidity ratios (ie. current ratio) are taken from financial statements such as: balance sheet, income statement and the statement of cash flow.

Early warning systems can spot liquidity risks by looking at both historical and projected financials. A low liquidity ratio could signal the company is suffering financial troubles.

However, a very high liquidity ratio isn't good either; it may indicate that the company is too focused on liquidity to the detriment of efficiently utilizing capital to grow and expand its business. According to the calculated values of the liquidity ratios the appropriate actions can be suggested by the system. A company can improve its liquidity ratios by raising the value of its current assets, reducing current liabil- 
TABLE II.

EXAMPLES OF WEBSITES OFFERING RESTFUL WEB SERVICES IN FINANCIAL DOMAIN

\begin{tabular}{|l|l|}
\hline URL & Description \\
\hline $\begin{array}{l}\text { http://www.bloomberg.com/c } \\
\text { ompany/ }\end{array}$ & $\begin{array}{l}\text { The established service provides free, unrestricted access to raw data for customers for its financial market } \\
\text { information. The same publish/subscribe and request/response interactions available via its proprietary interface } \\
\text { can be accessed via API. This functionality gives access to data on current market trades, either real-time or } \\
\text { delayed, along with reference data on reference data, historical information, and records of intraday trading. }\end{array}$ \\
\hline $\begin{array}{l}\text { http://www.gaincapital.com/1 } \\
\text { iquidity-api-trading.shtml }\end{array}$ & Capital offers a full range of CFD markets including indices, commodities, FX, bonds, interest rates and equities \\
\hline https://angel.co/ & $\begin{array}{l}\text { AngelList is a community of startups and investors with the goal of making fund raising efficient. Angel investors } \\
\text { are listed along with contact information which startups can use to set up introductions. The AngelList API } \\
\text { provides developers with a RESTful interface to the AngelList data set. Data includes followers, reviews, startups } \\
\text { and more. Responses are formatted in JSON and JSONP. }\end{array}$ \\
\hline $\begin{array}{l}\text { https://api.mattermark.com/ } \\
\text { The Mattermark REST API allows to query expansive dataset of companies. It gives access to all data available } \\
\text { by website interface, as well as the time series data about companies. It offers access to companies' profiles, } \\
\text { investors and funding events. }\end{array}$ \\
\hline $\begin{array}{l}\text { https://www.lendingclub.co } \\
\text { m/developers/api- } \\
\text { overview.action }\end{array}$ & \begin{tabular}{l} 
Offers REST API for services such as: personal and business loans and investing. \\
\hline
\end{tabular}
\end{tabular}

ities by paying off debt, or negotiating delayed payments to creditors. There are many solutions for resolving liquidity problem that can be suggested by the system and appropriate Web services can be found.

The procedure of the system's functioning contains the following steps:

a) an overall assessment of the firm's financial condition,

b) referring to the financial early-warning ontology to find possible corrective and preventive actions,

c) browsing available and relevant Web services to find sources of financing (such as: credits, new investors, business angels etc.),

d) analysis of risk and costs of the alternatives,

e) presenting the simulations to the manager,

f) successive, iterative searching of the alternatives of the highest efficiency.

Over the last few years, RESTful Web services have become popular due to their simplicity in publishing and consuming Web resources and better alignment with modern Web applications. The examples of sites offering Web services in financial domain are presented in Table II.

The proposed solution for supporting financial decision making by exploiting Web services works in the Web environment and can also be exposed as a compound sem-antic Web service (i.e. in the cloud). The main challenge in building the platform is to find and provide the relevant Web services from many providers which could be useful in financial decision making. It is also very important to maintain and update the base whenever there are important changes brought by the service providers considering the service parameters and when new services and providers appear on the market.

The second challenge is semantic description of the Web services which on one hand should be matched with the concepts from financial ontology and on the other hand their technical details, input and output and parameters should be identified and described semantically.

It is worth to notice that there are attempts to unify access to Web Services particularly facing the problem of the lack of the registers of RESTful services. The solutions (for ex- ample iServe [42] are not domain-oriented and do not offer ontological support for matching the services with business process models. There is no Web platform that would offer a full range of specific services for supporting managers in taking financial decisions. There are many applications, which let managers to monitor financial KPIs and visualize them in the form of manager's cockpit. The detailed review of the solutions was presented in [43]. Although many functions were offered, none of them was integrated with external web services to support managers in taking actions by suggesting specific services offered by banks and other financial institutions.

\section{V.CONCLUSION}

Web services are currently a preferred way to architect and provide complex services, there are many technical considerations in the literature regarding this field and many competing standards arise. The usability and efficiency of the proposed platform to a large degree depends on available Web services exposed by financial institutions therefore there is a challenge of integrating services from many providers, where the semantic Web technology can help.

There are several limitations while using financial measures, which can be addressed by semantic Web services. Financial ratios highlight the situation of the enterprise but their interpretation is needed to give answers to problems. Financial ontologies can be valuable source of expertise for managers in this field. The measures of the economic condition of the company are as accurate as the data used to calculate them. Semantic Web services offered by trusted companies can provide data of good quality that can be used for industry-specific benchmarks.

At the moment, the development of the described platform is on its first, conceptual stage. Although, it should be noted that some ontologies have already been elaborated and applied in the area of early warning and decision support system InKoM [44]. Ontologies build with one purpose in mind, such as early warning system, can be reused in other areas because the knowledge codified this way is independent of technical solutions. 
The main challenge and future work direction in the described approach is the segmental breakdown of financial policies and procedures into granular steps that can be performed by relevant Web services. Current work is also directed towards finding the most efficient solution for the Web services discovery and specification.

\section{ACKNOWLEDGMENT}

First of all I would like to express my gratitude to Jerzy Korczak, Wroclaw University of Economics, Poland, for providing access to the financial ontologies developed within the project InKoM and his valuable comments to the draft of the paper. I also thank Bogdan Franczyk, University of Leipzig and Maciej Pondel, Intratic Software Solutions for Business, for comments and suggestions on the technical details of RESTful Web services. I thank Jolanta Chluska, Czestochowa University of Technology, and Piotr Oleksyk Wroclaw University of Economics for consultation on the financial issues.

\section{REFERENCES}

[1] C. Olszak, "Wybrane technologie informatyczne w doskonaleniu rozwoju systemów Business Intelligence," in Zastosowania systemów informatycznych zarzqdzania, Problemy Zarzadzania, special issue, W. Chmielarz, J. Kisielnicki, T. Parys and O. Szumski, Eds. Wydawnictwo Naukowe Wydziału Zarządzania Uniwersytetu Warszawskiego, 2011, pp. 85-96.

[2] J. Korczak and H. Dudycz, "Intelligent dashboard for SME managers. Architecture and functions," in Proceedings of the Federated Conference on Computer Science and Information Systems FedCSIS 2012, pp. 1003-1007.

[3] P. Gibcus, P.A.M. Vermeulen and J.P.J Jong, "Strategic decision making in small firms: a taxonomy of small business owners," in International Journal of Entrepreneurship and Small Business, vol. 7, no. 1, 2009, pp. 74-91.

[4] J. A. Howard, "Financial decision-making: the roles of intuition, heuristics and impulses," in Journal of Modern Accounting and Auditing, Vol. 9, no. 12, 2013, pp. 1596-1610.

[5] E. Dane, K. V.V. Rockmann and M.G. Pratt, "When dhould I trust my gut? Linking domain expertise to intuitive decision-making Effectiveness," in Organizational Behavior and Human Decision Processes, 119, no. 2, 2012, pp. 187-94.

[6] E. Blomqvist, "The use of semantic Web technologies for decision support - a survey," in Semantic Web Journal, 5(3), IOS Press 2014, pp. 177-201, http://www.semantic-web-journal.net/sites/default/files/ swj299_0.pdf

[7] D. Fensel, H. Lausen, A. Polleres, J. De Bruijn, M. Stollberg, D. Roman and J. Domingue, Enabling Semantic Web Services: Web Service Modeling Ontology, Springer 2006.

[8] M. Ouzzani and A. Bouguettaya, Semantic Web Services for Web Databases, Springer Science+Business Media, LLC, 2011, DOI 10.1007/978-1-4614-1644-97

[9] T. Bellwood et al., "Universal Description, Discovery and Integration specification (UDDI) 3.0". 2002, http://uddi.org/pubs/uddi-v3.00published-20020719.htm.

[10] R. Chinnici, et al., "Web Services Description Language (WSDL) 1.2", 2007, http://www.w3.org/TR/wsdl/.

[11] D. Box et al. "Simple Object Access Protocol (SOAP) 1.1". 2001, http://www.w3.org/TR/SOAP/.

[12] R.T. Fielding, Architectural Styles and the Design of Network-Based Software Architectures, PhD dissertation, Department of Information and Computer Science, University of California, Irvine, 2000, https://www.ics.uci.edu/ fielding/pubs/dissertation/rest_arch_style.ht $\mathrm{m}$

[13] Oasis, "Web Services Business Process Execution Language Version 2.0," 2007, http://docs.oasis-open.org/wsbpel/2.0/OS/wsbpel-v2.0OS.pdf

[14] DAML, "DAML-S: Semantic Markup for Web Services," 2003, http://www.daml.org/services/daml-s/0.9/daml-s.html
[15] J. Cardoso and A.P. Sheth, "Introduction to semantic Web services and Web process composition". In Proc. of First Intl Workshop on Semantic Web Services and Web Process Composition (SWSWPC'04), San Diego, CA, USA 2004.

[16] C. Petrie. T. Margaria, H. Lausen and M. Zaremba eds., Semantic Web Services Challenge, Results from the First Year, Springer Science-Business Media, LLC, 2009.

[17] A. Karray, R. Teyeb and M. Ben Jemaa, "A heuristic approach for Web-service discovery and selection," in International Journal of Computer Science \& Information Technology, 5(2) 2013.

[18] A. Carenini, et al.,"Semantic Web service discovery and delection: a test bed scenario," in Sixth International Workshop on Evaluation of Ontology-Based Tools and the Semantic Web Service Challenge (EON-SWSCO8), CEUR Workshop Proceedings, vol. 359, R GarcíaCastro, A. Gómez-Pérez, C.J. Petrie, E. Delia Valle, U. Ktister, M. Zaremba and O. Shafiq eds., RWTH Aachen, Aachen, 2008, http://sunsitc.informatik.rwth-aachen.de/Publications/ CEURWS/Vol-359/

[19] R. Siedlecki, "Prognozowanie trudności finansowych przedsiębiorstw z wykorzystaniem miary rozwoju Hellwiga," in Prace Naukowe Uniwersytetu Ekonomicznego we Wrockawiu nr 323, 2013, pp. 308318.

[20] J. Collins, W, Ketter and M. Gini, "Flexible decision support in dynamic inter-organisational networks," in European Journal of Information Systems 19(4), 2010 pp. 436-448

[21] Sage, "Sage Canadian Small Business Financial Literacy Survey" Canadian-Small-Business-Financial-Literacy-Survey 2012, http://www.sage.com/na/ /media/site/sagena/documents/surveys/Sag e-Canadian-Small-Business-Financial-Literacy-Survey

[22] F. K. Andoh and J. Nunoo, "Sustaining Small and Medium Enterprises through Financial Service Utilization: Does Financial Literacy Matter?", University of Cape Coast, Ghana 2012, $\mathrm{http}: / / \mathrm{www} . u c l a n . a c . u k /$ research/explore/groups/assets/igfd sustainin g small and medium_enterprises_through_financial_service_utilizat ion.pdf

[23] N. Plakalović, "Financial literacy of SMEs managers", Make Learn, International Conference 2011, http://www.toknowpress.net/ISBN/ 978-961-6914-13-0/papers/ML15-086.pdf

[24] S. Srirama, M. Jarke and W. Prinz, Mobile host: "A feasibility analysis of mobile Web," in The 4th International Workshop on Ubiquitous Mobile Information and Collaboration Systems, 2006, pp. 942-953.

[25] J. Fuller, M. Krishnan, K.Swenson and J. Ricker, "Oasis asynchronous service access protocol (asap)," May 18 2005, http://www.oasisopen. org/committees/documents.php?wg_abbrev=asap

[26] J. Bergstra and M. Burgess, eds., The Handbook of Network and System Administration, Elsevier, 2007.

[27] L. Moreau, S. Miles, J. Papay, K. Decker and T. Payne, "Publishing Semantic Descriptions of Services", Proceedings of Global Grid Forum 9, Chicago IL, USA, 2003.

[28] S. Sowmya Kamath, V. S. Ananthanarayana, "Semantic Web Services Discovery, Selection and Composition Techniques". Third International Conference on Computer Science \& Information Technology, 2013 doi: 10.5121/csit.2013.3616

[29] L. Guo, Y.H. Chen-Burger, and D. Robertson, "Mapping a business process model to a semantic Web dervice model," in Proceedings of the 2nd IEEE International Conference on Web Services, 2004, pp. 746-749.

[30] Oasis, "UDDI Spec Technical Committee Draft", 2004 http://www. uddi.org/pubs/uddi v3.htm\# Toc85907967

[31] D. Mukhopadhyay, A. Chougule, "A survey on Web service discovery approaches," in Advances in Computer Science, Engineering \& Applications, D.C. Wyld, J. Zizka and D. Nagamalai, eds., AISC, vol. 166, Springer, Heidelberg 2012, pp. 1001-1012

[32] R. Douence R. et al., "Compositional Evolution of Secure Services using Aspects", 2010 http://cessa.gforge.inria.fr/lib/exe/fetch.php? media=publications:d1-1.pdf

[33] Y. Lee, "Semantic matching and resource discovery algorithms for RESTful Web services," in International Journal of Innovative Research in Computer and Communication Engineering Vol. 1, Issue 9, November 2013, http://www.ijircce.com/upload/2013/november/ 0A Semantic.pdf

[34] J. Recker, J. Mendling, "On the translation between BPMN and BPEL: conceptual mismatch between process modeling languages," in CAiSE 2006 Workshop Proceedings - Eleventh International Workshop on EMMS AD. Luxembourg. June 5-6, pp. 521-532, 2006 
[35] S. Dustdar and F. Li, eds., Service Engineering: European Research Results, 2011 Springer-Verlag/Wien, 2011.

[36] E. Christensen, F. Curbera, G. Meredith and S. Weerawarana, "Web Services Description Language (WSDL) 1.1”' 2001, https://www.w3.org/TR/wsdl

[37] J. Nitzsche, T. van Lessen, D. Karastoyanova and F. Leymann, "BPEL for semantic Web services (BPEL4SWS)," in Proceedings of the 3rd International Workshop on Agents and Web Services in Distributed Environments AWeSome'07 -- On the Move to Meaningful Internet Systems: OTM 2007 Workshops', Springer-Verlag, 2007, pp. 179188

[38] D. Martin, et al, "OWL-S: Semantic Markup for Web Services W3C" Member Submission 22 November 2004, http://www.w3.org/ Submission/OWL-S

[39] J. De Bruijn, et al., "Relationship of WSMO to other relevant technologies" W3C Member Submission 3 June 2005 http://www. w3.org/Submission/WSMO-related/\#owl-s
[40] S. Galizia, A. Gugliotta, C. Pedrinaci and J. Domingue, "Applying semantic web services," in 4th Workshop on Semantic Web Applications and Perspectives (SWAP 2007), Bali, Italy 2007 http://ceur-ws.org/Vol-314/48.pdf

[41] K.A. Horcher, Essentials of Managing Treasury, New Jersey, John Wiley \& Sons 2005.

[42] Datahub, "iServe: Linked Services Registry" 2014 http://datahub.io/ dataset/iserve

[43] P. Ziuziański and M. Furmankiewicz M., "Kokpit menedżerski jako narzędzie do wizualizacji danych w kontekście zarządzania wiedzą w organizacji," in Zeszyty Naukowe Politechniki Białostockiej. Ekonomia i Zarzqdzanie, z. 7(1), 2015, pp. 44-60.

[44] J. Korczak, H. Dudycz, M. Dyczkowski, "Inteligentny kokpit menedżerski jako innowacyjny system wspomagajacy zarzadzanie w MŚP," in Informatyka Ekonomiczna, 1(31), 2014, pp. 288-303. 